\title{
Optimization of Diesel Engine Based on Digital Design Technology and Finite Element Simulation Analysis Weng Lingxiao ${ }^{1}$ and Ren Jiachao $^{2}$
}

\author{
(1.NanChang Institute of Science and Technology, Nanchang,330108; Jiangxi Engineering \\ Laboratory of Digital Manufacturing for Automobile Parts; 2. AnHui QuanChai Engine CO.,LTD)
}

Keywords: Digital Design Technology; Diesel Engine; Simulation, Finite Element

\begin{abstract}
With the improvement of the enhancement level of vehicle power and the demand for high reliability power required by the equipment, the contradiction between high performance, high compactness and high reliability has become increasingly prominent. The diesel engine is designed as an organic combination of several subsystems by using the overall design theory and methods (including integration methods, simulation methods, and comprehensive comparison methods, etc., with top-level planning, system coverage, comprehensive coordination, trade-offs, and iterative iterations, to find the overall optimal solution. The overall design process is focused on solving the contradiction between the application of advanced technology and the realization of engineering. It adopts advanced methods and its supporting technologies to improve product structure, and increase the understanding of product performance, so that its products have the shortest development cycle, the lowest cost and the highest performance. The creation of a digital prototype is a top-down, all-encompassing overall design.
\end{abstract}

\section{Introduction}

The diesel engine uses a modern product design method based on digital design technology depending on knowledge engineering and multidisciplinary technologies to formulate impeccable modern processes and specifications, and carry out the structural optimization design for the key components such as diesel engine crankshaft, connecting rod, camshaft, engine block and cylinder lid, as well as the optimized matching of intake and exhaust, cooling and lubrication systems. The study finds that it can save development time and costs, improve the quality and reliability of diesel engine products, complete the transition from relying on personal "experience design" to "predictive design" based on the company's core capability as well as enable the vehicle power to gradually develop toward the "successful" direction by the simulation, analysis and product optimization of the digital models, predicting and evaluating the performance of products in advance and reducing research work at the prototype stage before the establishment of physical prototypes.

\section{Digital Design Technology}

The research core of the digital design technology is to use computer-aided analysis techniques to perform the dynamics and kinematics analysis of mechanical systems. Kinematics focuses on the analysis of the movability and trajectories of product mechanisms. Through kinetic analysis, the dynamic reaction of each kinematic pair in the process of motion for the product mechanism is the basis of the strength analysis. The complete diesel digital prototype is a true digital simulation of the final product. It can perform engineering analysis and optimization, determine the optimal design structure and parameter values, and obtain the best overall performance.

(1) Motion analysis. Verify the accuracy of the physical position of crankshaft linkage mechanism, transmission mechanism and valve distribution mechanism in the process of space motion, analyze the movement clearance, movement coordination, etc. during the movement process of each mechanism, and perform numerical analysis of displacement, speed and acceleration, etc. for the geometric elements or movement particles during the movement process.

(2) Performance parameter matching analysis. Combining the test data, digital analysis tools 
are used to carry out matching analysis of various performance parameters in the diesel engine working process, and perform parameter matching of subsystems such as intake and exhaust, cooling, lubrication, and oil supply.

(3) Spatial structure analysis. Implement interference check, clearance check, key section analysis and measurement space analysis, so that designers can intuitively learn about the problems in the prototype, such as calculate the gap changes under different conditions of the diesel engine by setting the thermal physical parameters of the diesel engine parts.

(4) Assembly analysis. In accordance with the assembly process, fixture of physical prototype, its assembly process was simulated, the parts and theirassembly fixture were detected dynamically in the assembly and dismantling process for interference, and assembly clearance was analyzed; the structure of the diesel engine and its space requirements in the assembly process were analyzed; the assembly sequence and assembly path were verified to provide data support for defining, predicting, and analyzing part manufacturing tolerances and technical requirements.

(5) Process assessment. The structure manufacturability of digital prototyping was evaluated, including the coordination of prototypes on the bench, Design for Manufacturing (DFM), Design for Assembly (DFA), and Tolerance Analysis.

\section{Optimization Design Process of Diesel Engine}

Optimization design generally includes overall plan optimization and design parameter optimization. The main task of the overall solution optimization (optimization of the overall layout, structure or system model) is to establish and process the knowledge model, and optimize the non-numerical knowledge of complex mechanical product design schemes based on expert systems, to get the reasonable design through the inference with artificial intelligence, expert systems, and fuzzy judgments. The main task of design parameter optimization (product part structure, process parameter optimization, etc.) is to establish a correct optimization mathematical model and try to obtain the optimal value. The joint optimization process of diesel engine design parameters $\mathrm{CAD} / \mathrm{CAE}$ is shown in Figure 1. It usually needs to complete through the following steps, and the degree of automation is getting higher and higher.

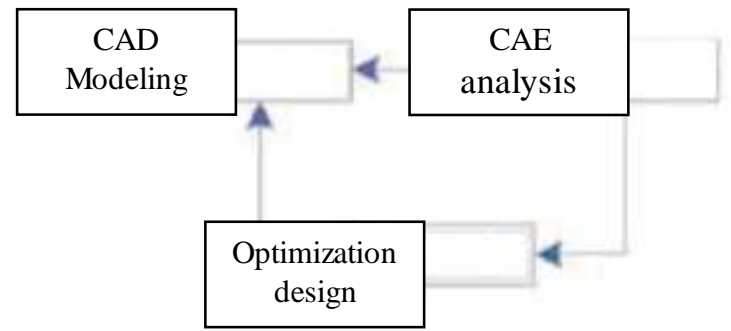

Fig. 1 The CAD/CAE joint optimization process for the design parameters of the oil machine

1. Preparatory phase

The product structure scheme was established approximately according to the actual problem. The type of simulation problem, the degree of simplification of the simulation model, and the load and constraint conditions of the simulation model should be determined based on the simulation mission objectives. The simulation model is a digital model established based on specific simulation tasks, including geometric model, boundary conditions, initial conditions, and calculation settings.

2. Parametric modeling

The actual problem of mechanical design is abstracted as the mathematical model of the optimal design, and the data (design variables) to be involved in the optimization is defined as the model parameters, which makes it possible to modify the model later by the software. The mathematical model should be established based on the requirements of simulation task, to determine the calculation domain and boundary conditions, and set the calculation parameters and so on.

3. Solution

The program applying the optimization calculation method solves the parametric CAD model on 
the computer. For specific tasks, calculation and solution are submitted to the analysis software by the way of command stream or file mode, and the calculation process is monitored by command stream or window interaction.

\section{Post processing}

The simulation results are processed and analyzed. The post-processing graphics function of FEA software is used to display the temperature distribution map, deformation map, and stress distribution map, from which the maximum temperature, maximum deformation, maximum stress, and minimum life span are found. They are evaluated according to the corresponding criteria and summarized in the form of pictures or tables, and simulation analysis reports are provided based on application requirements.

\section{Multidisciplinary Simulation Analysis of Diesel Engine}

The development of diesel engine based on digital prototyping technology requires the analysis of diesel engine thermodynamics, kinematics analysis and dynamic analysis of the crank linkage mechanism at the conceptual design stage, as well as the diesel engine stiffness analysis, vibration and noise analysis, performance analysis and reliability analysis at the design stage of the plan. Furthermore, the analysis of internal cooling liquids and external circulation, analysis of the flow field of lubricating fluids, and analysis of air flow fields in intake and exhaust systems are also required. The diesel engine structural simulation refers to the process of obtaining the displacement, stress, strain, etc. under the working state of the diesel engine through the numerical calculation method using the whole or part physics and mathematical model of the diesel engine. The key components of the diesel engine (taking the cylinder head as an example) are generally divided into rigid strength analysis, modal analysis, thermal analysis and fatigue analysis.

(1) Rigid strength analysis (also called static analysis) includes linear and nonlinear analysis to solve the stress, displacement and deformation of the structure under the static load (or quasi-static load) .

(2) Modal analysis is used to determine the intrinsic vibration characteristics of a structure or component, that is, the natural frequency and mode shape of the structure. It can also be used as a starting point for other dynamic analysis problems, including transient dynamic analysis, harmonic response analysis, and spectrum analysis and so on. For the integrated cylinder lid, it is necessary to analyze the basic dynamic characteristics such as the free modal frequency and vibration mode, and provide basic data for dynamic response analysis and dynamic characteristics optimization design of the cylinder lid.

(3) Thermal analysis mainly refers to calculating the given heat transfer boundary conditions or the heat transfer boundary conditions of empirical formula based on the flow field, and the temperature field of the airframe and cylinder lid is analyzed to provide thermal boundary conditions for other structural simulation analysis. The temperature and thermal stress distribution of the cylinder lid under the influence of high temperature gas, coolant and external environment needs to be analyzed. The structural strength of the cylinder lid under the combined action of mechanical load and temperature load is evaluated to provide temperature and stress fields for fatigue simulation.

\section{Conclusion}

Modern design and optimization technologies have effectively promoted the in-depth development of system integration and matching research, and significantly improved the optimal matching between the power density of power plant and system assembly. The high power density diesel engine is a high-compact overall structure design based on high power. High-stress materials are used for integrated system integration design, reducing ineffective space, achieving the largest power, the smallest volume, the lightest quality and the lowest cost. Through the optimization design of integration, serialization and modularization for diesel engine components, the high compactness, and high mobility requirements of the whole equipment are met, so that the unit 
volume power, unit displacement power of diesel engine reach to the highest level, speeding up the product development cycle, reducing $\mathrm{R} \& \mathrm{D}$ costs and technology risks, and improving market competitiveness.

\section{Acknowledgement}

Project funding: Project at Nanchang institute of science and technology (No.SZZX-17-03)\& Key National Development Projects(No. 2016YFD0700801)

\section{References}

[1] Wu J F, Wang H N, He X D, et al. Ergonomics Optimization of Human-Product Contact Surface Design Based on Stochastic Finite Element[J]. Advanced Materials Research, 2010, 102-104:160-164.

[2] Zuo X M, Xu Z M. Thermal Analysis and Optimization of Plant-Fiber and Starch Dishware Mould Based on ANSYS[J]. Advanced Materials Research, 2010, 102-104:125-129.

[3] Zhang Y K, Xiao L J, Yang J R. Finite Element Simulation Analysis of Hydraulic Support Based on Virtual Prototype Technology[J]. Applied Mechanics \& Materials, 2012, 217-219:1393-1396.

[4] Du Y P, Hu G L, Dong F. Study on Structural Design and Optimization Analysis for 2V80 Engine Piston Based on Finite Element Analysis[J]. Advanced Materials Research, 2013, 753-755:1188-1191.

[5] Sun H J, Yin G F, Mi L, et al. Study on the Design and Analysis of Spindle System of NC Machine Tools Based on Digital Design[J]. Key Engineering Materials, 2012, 522:668-672.

[6] Liu L M, Shan Z D, Liu F. Finite Element Analysis and Optimization for the Structure of NC Forming Machine[J]. Advanced Materials Research, 2014, 945-949:166-173.

[7] Wang J, Feng J, Zhang S. Optimization of pipe assembly path planning based on finite element simulation analysis[J]. Science \& Technology Innovation Herald, 2015.

[8] Luo H F, Wang J C, Lei J, et al. Research on Crack of Cylinder Bracket Based on Numerical Simulation and Finite Element Analysis[J]. Advanced Materials Research, 2014, 940:236-240.

[9] Cheng Y N, An S, Liu X L, et al. Experimental Study and Simulation Analysis on Ball-End Cutter Milling Titanium Alloy Based on the Geometric Parameters Optimization[J]. Key Engineering Materials, 2014, 589-590:427-432.

[10] Zhou H, Hou K, Pan H, et al. Structure optimization of eddy current sensor based on the finite element simulation[J]. Electronic Measurement Technology, 2016.

[11] Dai W, Lian Y, Xiao J, et al. Analysis and optimization of plunger necking-in process based on finite element simulation[C]// First International Conference on Reliability Systems Engineering. IEEE, 2016:1-6.

[12] Yang Y, Zou J. Optimization Design of Longitudinal-torsional Ultrasonic Transducer Based on Finite Element Model[J]. Journal of Dongguan University of Technology, 2017. 